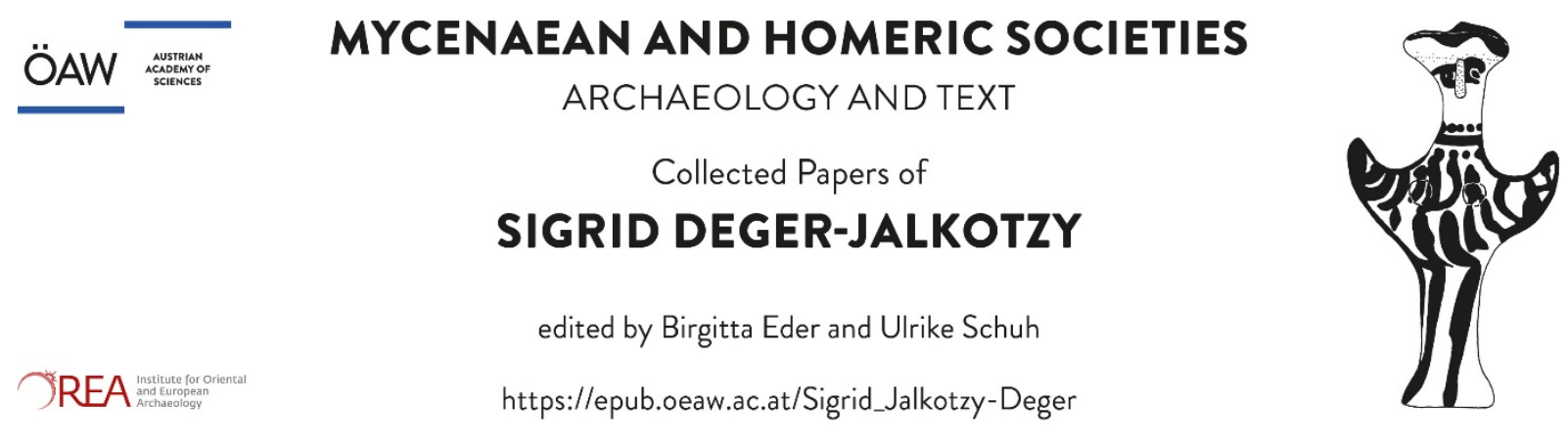

34.

S. Deger-Jalkotzy

\title{
Dunkle Jahrhunderte
}

in: Der Neue Pauly, Enzyklopädie der Antike 3 (Stuttgart - Weimar 1997) 838-843

(C) J.B. Metzler [Brill] https://referenceworks.brillonline.com/browse/brill-s-new-pauly mit freundlicher Genehmigung / with kind permission

Dieses Dokument darf ausschließlich für wissenschaftliche Zwecke genutzt werden (Lizenz CC BY-NC-ND), gewerbliche Nutzung wird urheberrechtlich verfolgt.

This document is for scientific use only (license CC BY-NC-ND), commercial use of copyrighted material will be prosecuted. 


\section{(DNP)}

Fachgebietsherausgeber

Prof. Dr. Gerhard Binder, Bochum

Kulturgeschichte

Prof. Dr. Hubert Cancik, Tübingen

Geschäftsführender Herausgeber

Prof. Dr. Walter Eder, Bochum

Alte Geschichte; Rezeption: Wissenschaftsgeschichte

Prof. Dr. Burkhard Fehr, Hamburg

Klassische Archäologıe (antike Alltags-,

Architektur- und Kunstgeschichte)

Prof. Dr. Bernhard Forssman, Erlangen

Sprachwissenschaft; Rezeption: Sprachwissenschaft

Prof. Dr. Fritz Graf, Basel

Religion und Mythologie; Rezeption: Religion

PD Dr. Hans Christian Günther, Freiburg

Textwissenschaft

Prof. Dr. Berthold Hinz, Kassel

Rezeption: Kunst und Architektur

Dr. Christoph Höcker, Hamburg

Klassische Archäologie (antike Alltags-,

Architektur- und Kunstgeschichte)

Prof. Dr. Christian Hünemörder, Hamburg

Naturwissenschaften und Technik; Rezeption:

Naturvissenschaften

Dr. Margarita Kranz, Berlin

Rezeption: Philosophie

Prof. Dr. André Laks, Lille

Philosophie

Prof. Dr. Manfred Landfester, Gießen

Geschäftsführender Herausgeber: Rezeptions- und

Wissenschaftsgeschichte; Rezeption: Wissen-

schaftsgeschichte

Prof. Dr. Maria Moog-Grünewald, Tübingen Rezeption: Komparatistik und Literatur

Prof. Dr. Dr. Glenn W. Most, Heidelberg

Griechische Philologie
Prof. Dr. Beat Näf, Zürich

Rezeption: Staatstheorie und Politik

Dr. Johannes Niehoff, Freiburg Judentum, östliches Christentum, byzantinische Kultur

Prof. Dr. Hans Jörg Nissen, Berlin Orientalistik

Prof. Dr. Vivian Nutton, London Medizin; Rezeption: Medizin

Prof. Dr. Eckart Olshausen, Stuttgart Historische Geographie

Prof. Dr. Filippo Ranieri, Saarbrücken

Rezeption: Rechtsgeschichte

Prof. Dr. Johannes Renger, Berlin Orientalistik; Rezeption: Alter Orient

Prof. Dr. Volker Riedel, Jena

Rezeption: Erziehungswesen, Länder (II)

Prof. Dr. Jörg Rüpke, Potsdam Lateinische Philologie, Rhetorik

Prof. Dr. Gottfried Schiemann, Tübingen Recht

Prof. Dr. Helmuth Schneider, Kassel Geschäftsführender Herausgeber; Sozialund Wirtschaftsgeschichte, Militärwesen; Wissensschaftsgeschichte

Dr. Frieder Zaminer, Berlin Musik; Rezeption: Musik

Prof. Dr. Bernhard Zimmermann, Freiburg Rezeption: Länder (I)

\section{Redaktion}

Beate Baumann Jochen Derlien Dr. Brigitte Egger

Christa Frateantonio Dr. Ingrid Hitz] Vera Sauer Dorothea Sigel Anne-Maria Wittke 


\section{DER NEUE PAULY}

\section{Enzyklopädie der Antike}

Herausgegeben

von Hubert Cancik und

Helmuth Schneider
Altertum

Band 3 Cl-Epi 


\section{Inhaltsverzeichnis}

Dic Dentsche Bibliothek - CIP-Einhcitsnufinahme

Der ncus Pauly : Enzyklopädie der Antike/hrsg. von Hubert Cancik und Helmuth Schneider. Stuttgart ; Weimar : Metzler, 1997

ISBN 3-476-01 $470-3$

NE: Cancik, Hubert [Hrsg.]

Bd. 3. Cl-Epi - 1997

ISBN 3-476-OI 473-8

Gedruckt auf chlorfrei gebleichtem, säurefreiem und alterungsbeständigem Papier

Dieses Werk einschließlich aller seiner Teile ist urheberrechtlich geschützt. Jede Vernvertung außerhalb der engen Grenzen des Urheberrechtsgesetzes ist ohne Zustimmung des Verlages unzulässig und strafbar. Das gilt insbesondere für Vervielfältigungen, Übersetzungen, Mikroverfilmungen und die Einspeicherung und Verarbeitung in elektronischen Systemen.

ISBN 3-476-0I 470-3 (Gesamtwerk)

ISBN 3-476-0 1 473-8 (Band 3 Cl-Epi)

(C) 1997 J.B. Metzlersche Verlagsbuchhandlung und Carl Ernst Poeschel Verlag $\mathrm{GmbH}$ in Stuttgart

Typographie und Ausstattung: Brigitte und Hans Peter Willberg Grafik und Typographie der Karten:

Richard Szydlak

Abbildungen: Günter Müller

Satz: pagina $\mathrm{GmbH}$, Tübingen

Gesamtfertigung: Franz Spiegel Buch

GmbH, Ulm

Printed in Germany

Verlag J. B. Metzler Stuttgart · Weimar
Hinweise für die Benutzung

Transkriptionstabellen

VI

Erweitertes Abkürzungsverzeichnis

VIII

Karten- und Abbildungsverzeichnis

XLV

Verzeichnis der Autoren

XLVIII

Verzeichnis der Übersetzer

Mitarbeiter in den FG-Redaktionen

Lemmata

Corrigenda zu Band I und 2 
Dumnorix (Dubnoreix; keltisches Namenskomp. "Weltkönig" [1. 85-86]). Fürst der Haedui, Bruder des Diviciacus und Schwiegersohn des Orgetorix, mit dem er die Eroberung ganz Galliens geplant haben soll (Caes. Gall. I,3). D. war der mächtigste Mann im Stamm, be$\mathrm{sa} ß$ eine private Reitertruppe und verfügte zudem durch Geschenke und Heiratspolitik über großen Einfluß bei fremden Stämmen, so daß er im J. 58 v. Chr. den Helvetii zum Durchzug durch das Sequanergebiet verhelfen konnte (Caes. Gall. I, 18,3-19,4). Caesar verdächtigte ihn der Konspiration, ließ ihn aber nur beobachten, um den Bund mit den Haedui nicht zu gefährden (Caes. Gall. I, 1 8,10-20,6). Offenbar hat sich D. in den folgenden Jahren ruhig verhalten, als er aber 54 v. Chr. Caesar auf dessen zweiten Britannienfeldzug als Geisel begleiten sollte, versuchte er zunächst, sich der Überfahrt mit allen Mitteln zu entziehen und auch andere zum Bleiben zu bewegen. Schließlich wurde er bei einem Fluchtversuch von röm. Truppen verfolgt und getötet (Caes. Gall. 5,6-7). Seine Bed. vor der Ankunft Caesars wird durch zahlreiche Silbermünzen mit seinem Namen unterstrichen [2. 429-43 I].

$\rightarrow$ Diviciacus; Haedui; Helvetii; Orgetorix

1 Evans 2 B. Colbert de Beaulieu s. Diviciacus [1]

B. Kremer, Das Bild der Kelten bis in augusteische Zeit, 1994, 219-239.

Dumnovellaunus (Dubnovellaunus; keltisches $\mathrm{Na}-$ menskomp. "der die Welt sieht«? [1. 196-I97; 272277]). König einiger Stämme im östl. Kent um I 5 v.- ca. I 5 n. Chr. [2. Nr. 275-29i A]. Um die Zeitenwende eroberte er das Gebiet der Trinovantes nördl. der Themse und etablierte sich in Camulodunum. Ca. Io n. Chr wurde er von dort durch Cunobellinus vertrieben. Wohl aus diesem Anlaß ist er zu Augustus geflohen, der ihn in seinem Tatenbericht erwähnt (R. Gest. div. Aug. cap. 32).

$\rightarrow$ Camulodunum; Cunobellinus

1 Evans 2 R.P. Mack, The Coinage of Ancient Britain, 1964, 95, 103.

W.SP.

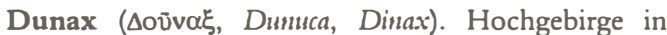
Westthrakien, wahrscheinlich das Rila-Gebirge in Bulgarien (Ptol. 34,10,15 = Strab. 4,6,12; Liv. 40,58,2: Kampf der Thrakes mit den Bastarnae, 179 v.Chr.). Die gleichlautenden PN sind kaum mit diesem Namen zu verbinden.

I. v. B.

Dunius. L.D. Severus. Proconsul von Pontus-Bithynien unter Claudius.

PIR $^{2}$ D 207 - W. WEISER, in: ZPE 1997 (in Vorbereitung).

\section{Dunkle Jahrhunderte}

[1] (1 200-800 v. Chr.)

\section{A. Definition}

Der Begriff D.J., geprägt im angelsächsischen Sprachraum ("Dark Ages"), charakterisiert seit dem ausgehenden I9. Jh. die Zeit vom Untergang der myk. Paläste ca. I 200 bis zum Beginn der „Homerischen Zeit", also der früharcha. Periode Griechenlands im 8. Jh. v. Chr. Archäologisch umfassen die D.J. die Abschnitte Späthelladisch (SH) III C = Mykenisch III C (12. und frühes 1 I. Jh.), Submykenisch (frühes bis Mitte 1 I. Jh.), Protogeometrisch (PG; ab Mitte I I. Jh. bis ca. 900), Früh- und Mittelgeometrisch (FG, MG; 9. und frühes 8. Jh.). SH III C und Submyk. waren letzte Phase und Ausklang der myk. Kultur, PG, FG und MG gelten auch als die "Frühe Eisenzeit" Griechenlands. Die mit dem Begriff D. J. verbundene Vorstellung von kultureller Bedeutungslosigkeit, Verarmung, fehlender sozialer Differenzierung und Abbruch von Außenbeziehungen ist so nicht länger zu halten. Zwar sank nach dem Zusammenbruch der hochentwickelten myk. Palaststaaten und -kultur ( $\rightarrow$ Ägäische Koiné B.4) die griech. Kultur in nahezu allen Sektoren auf ein Niveau, das sich weder mit der myk. Hochkultur noch mit der Kultur ab 750 v. Chr. messen kann, doch läßt der neuere Forschungsstand durchaus schöpferische und zukunftsweisende Leistungen der Griechen auch in den D.J. erkennen. Schon im I I. Jh. zeichnen sich, als "Übergang von der Bronze- zur Eisenzeit" umschriebene, technologische Neuerungen ab, die es etwa erlauben, Großgefäße mit Dekor aus konzentrischen, mit Zirkel und Mehrfachpinsel gezogenen Kreisen und Halbkreisen herzustellen und der PG Vasenkunst Athens und Euboias den Kunstbegriff "Stil" zuzusprechen. Zudem formten sich in den D. J. die griech. Dialekte aus; mit der epischen Dichtung vom Typ der $\rightarrow$ "Oral Poetry" entstand die Grundlage für die homer. Großepik. Soziale Schichtung und Herrschaftsbildung sind ebenso erkennbar wie Schiffahrt und innerägäische Beziehungen; selbst Fernkontakte kamen, wenn auch reduziert, immer wieder zustande.

Der Begriff D. J. als Epochenbezeichnung ist jedoch vor allem durch den Verlust der Schrift und folglich das Fehlen jeglicher schriftlichen Tradition gerechtfertigt (die Linear B-Schrift verschwindet um I 200, das griech. Alphabet entsteht um 800). Verloren gingen auch Repräsentationsarchitektur, Festungs- und Brückenbau, sowie Kunstgattungen (die Kultstatuetten von Tiryns aus dem 12. und der Kentaur von LefkandiToumba aus dem Io. Jh. sind Ausnahmen), nur im Töpferei-und Waffenhandwerk überlebten die Fertigkeiten der Palastwerkstätten. Im polit. Bereich verschwanden die komplexe, zentralistisch-bürokratisch organisierte myk. Staatsordnung und das theokratische Königtum völlig. Überregionale Herrschaftsformen fehlen, erkennbar sind Organisationsformen in Kleinstaaten und Gemeinwesen, die auf Oikos, Verwandtschafts- und anderen Personalverbänden basieren. Im soz. und ökonomischem Bereich zeigt sich der Wandel auch darin, 


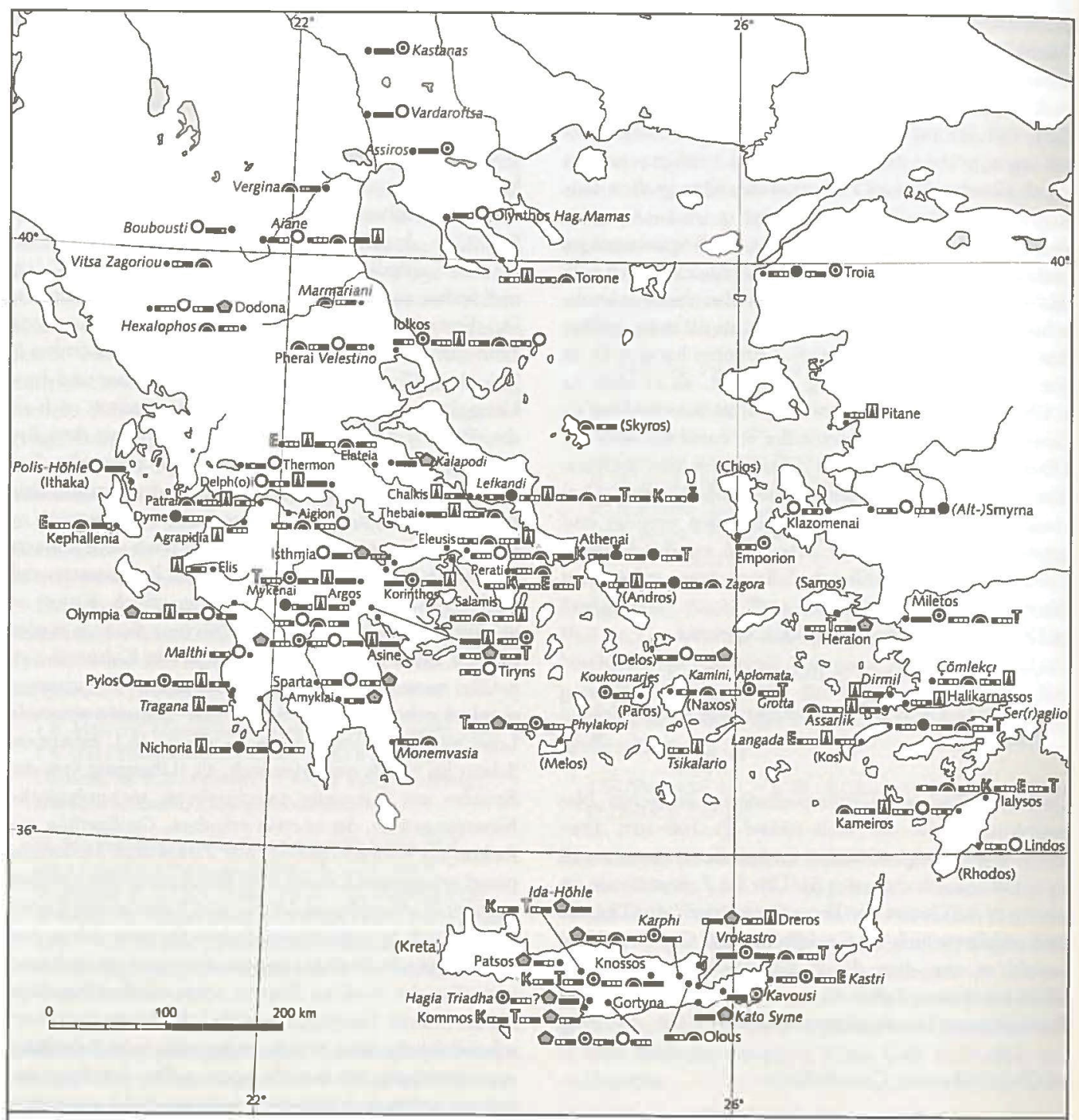

Griechenland und die Ägäis während der "Dunklen Jahrhunderte» (12. - 9. Jh.v.Chr.). Die wichtigsten Fundorte

\footnotetext{
- bedeutende Siedlung

○ $O$ Siedlung

(mit/ohne Architekturbefund)

คI Nekropole/Grab

- Heiligtum

3 Zentrum der Keramikproduktion

to Identifizierung unsicher
}

Chronologie:
Spãthelladisch (12./Anfang 11.Jh.)
Submyk./Submin.
(Anfang - Mitte 11.Jh.)
Protogeometrisch
(Mitte 11.Jh. - ca. 900 v.Chr.)
Geometrisch (Früh-, Mittelgeometrisch)
(9. - Anfang 8.Jh.)
$=$ Datierung, sicher, unsicher

Datierung, sicher, unsicher

\section{Importfundplatz: \\ T Orient \\ Kypros \\ E Italien, Europa}

Mykenai antiker Name

Çōmlekçı moderner Name

(Rhodos) Inselname 
$\mathrm{da} \beta$ die entsprechenden myk. Linear B-Termini meist nicht in den alphabetgriech. Sprachschatz gelangten.

B. I2. JAHRHUNDERT

Den Beginn von SH III C prägten Zerstörungen und Bevölkerungsbewegungen ("Ägäische Wanderung", "Seevölkerwanderung"), Siedlungen wurden an sichere Orte verlegt, doch blieb die Kultur trotz Neuerungen (Griffzungenschwerter, Gewandfibeln, "Barbarian"Keramik) myk. und zeigte sogar im späteren I2. Jh. ("Mittleres" SH III C) eine letzte Blüte in Siedlungen (etwa Mykene, Tiryns, Lefkandi-Xeropolis, ParosKoukounaries, Knossos, Kavousi), Kammergräbern (u. a. von Perati, Monemvasia, Elateia, Naxos, Kos, Rhodos, Mouliana) und Heiligtümern (Kalapodi, Phylakopi, Keos-Hagia Irini, Kato Syme, Ida-Höhle). Dabei entwickelte aber, anders als in der myk. Palastzeit, jede Region ihren eigenen, bes. in der Vasenmalerei sichtbaren Stil. In den Zentren kleiner Herrschaftsbereiche pflegte eine kriegerische Elite höfischen Lebensstil und stellte ihre Ideale auf Prunkvasen und in aufwendigen Kriegerbegräbnissen dar. Lebhafter Handels- und Kulturaustausch bestand zwischen dem Festland, Kreta und den Inseln. Fernkontakte reichten bis Zypern und Italien. Friede und Wohlstand dauerten teilweise bis ins Späte SH III C und endeten dann in neuerlichen Katastrophen.

\section{II. JAHRHUNDERT}

Das II. Jh. ist noch ungenügend erforscht, doch scheint die myk. Kultur regional sehr unterschiedlich ausgeklungen zu sein. Während in myk. Kemländern (Peloponnes, Kykladen, Dodekanes) Zerstörungen, die Aufgabe von Siedlungen und Bevölkerungsschwund zur Abwendung vom Mykenischen und einem Neubeginn führten, hielt sich in der sog. Peripherie (Mittelgriechenland, Makedonien, Thessalien, Ionische Inseln) myk. Erbe noch im Submyk. und PG. Auf Kreta pflegte die reiche Oberschicht der städt. Siedlung in Knosos Kontakte bis Zypern; der Osten Kretas blieb bis in PG Zeit minoisch (Karphi, Praisos, Vrokastro, Kastri); in Kalapodi, Kato Syme und in der Ida-Höhle zeigt sich Kultkontinuität. Zypern wurde durch Zuwanderungen aus Griechenland hellenisiert. Weite Teile Küstenkleinasiens wurden endgültig griechisch. Die ant. Tradition verbindet die demographischen Veränderungen zwar mit Migrationen griech. Ethnien $(\rightarrow$ Dorische Wanderung, $\rightarrow$ Ionische Wanderung), doch lassen sich neue Kulturelemente (wie Eisenwaffen und -objekte, lange Gewandnadelpaare, Einzelgräber, Leichenverbrennung, handgemalte alte Keramik) nicht mit spezifischen Bevölkerungsgruppen verbinden. Als prägend erwies sich im I I. Jh. die Schöpfung des PG Vasenstils in Athen.

\section{IO. JAHRHUNDERT}

Im Io. Jh. entstanden dann regionale PG Kulturkreise, unter denen Attika und Euboia führend waren. Neuere Grabungen dokumentieren erstaunlich unterschiedliche Formen polit. Organisation und wirtschaftlicher Tätigkeit: In Nichoria lebte eine Dorfgemeinschaft von
Viehzüchtern mit einfacher Kultur. Ein großes Apsidenhaus diente wohl dem Oberhaupt als Wohnsitz und zugleich als Versammlungsort für die Vornehmen. In Lefkandi zeigen die Gräberfelder ein ganz anderes Bild: Zahlreiche kostbare Beigaben des Toumba-Friedhofes belegen den Luxus und die Tatkraft einer unternehmerischen Aristokratie, die Objekte aus Zypern, Phönikien und Ägypten nach Euboia gelangen ließen. Die Keramik stand der Athens nicht nach und war stilbildend für Mittelgriechenland und die Kykladen. Das Monumentalgrab eines Kriegerfürsten (?) läßt an eine monarchische Struktur und an homer. Totenriten denken. In Athen und Knosos, bereits in PG Zeit urbanen Zentren, pflegten führende vornehme Familien weitreichende internationale Beziehungen. Auf Kreta belegen Funde in Knosos und im früheisenzeitlichen Tempel von Kommos ab dem Io. Jh. die Präsenz von Phönikern.

\section{E. 9. JAHRHUNDERT}

Auch im 9. Jh. bleiben Athen, Euboia und Knosos in Kultur und Handel führend. Die urbane Struktur verstärkt sich in Siedlungen wie Zagora (Andros), Emporion (Chios) oder Altsmyrna und leitet die spätere Trennung von Polis und "Stammesstaata ein. Neben einfache Wohnhäuser mit ovaler, apsidialer und rechteckiger Form treten Befestigungsbauten, nur selten auch Sakralbauten. In Kreta (Knosos-North Cemetery) zeigen sich schon im 9. Jh. Phänomene, die sonst im 8. Jh. auftreten: "nostalgische" Hinwendung zur brz. Vergangenheit, oriental. Einflüsse in de Kunst durch regelmäßigere Kontakte mit Zypern und der Levante sowie rasanter Anstieg der Bevölkerungszahlen.

Allg. Darstellungen: W.D.E. Coulson, The Greek Dark Ages, 1990 - V.R. D'A. Desborough, The Greek Dark Ages, $1972 \cdot$ F. Schachermeyr, Die ägäische Frühzeit, Bde. 3 und 4 , 1979/1980 - A.Snodgrass, The Dark Age of Greece, 1971 - C.-G. Styrenius,

Submycenaean Studies, $1967^{\circ}$ J.VANSChOONwinkel, L'Egée et la Mediterranée orientale à la fin du deuxième millénaire, 1991.

Kongresse, Regionale Studien, Einzelaspekte: P. A. Cartledge, Sparta and Lakonia, 1972 ' Ders., Early Lakedaimon, in: J.M. SANDERS (Hrsg.), Philolakon. FS H. Catling, 1992, 49-55 - R.W.V. Catuing, I.S. Lemos (Hrsg.), Lefkandi II/I, I990 - J. N. Coldstream, H.W. Catuing (Hrsg.), Knossos North Cemetery Early Greek Tombs, 1996 - W.D.E. Coulson, The Dark Age Pottery of Sparta, in: ABSA 80, 1985, 29-84 - Ders., The Protogeometric from Polis Reconsidered, in: ABSA 86, 1991, 43-64 F. Dakoronia, Spercheios Valley and the Adjacent Area in Late Bronze Age and Early Iron Age, in: La Thessalie, 1994 - S. Deger-JAlkotzy (Hrsg.),

Griechenland, die Ägäis und die Levante während der "Dark Ages« vom 1 2. bis zum 9. Jh.v. Chr., 1983 - Dies., Elateia (Phokis) und die frühe Gesch. der Griechen, in: Anzeiger der Österreichischen Akad. der Wiss. 127 (1990) 1991, 77-86 - B.EDER, Argolis, Lakonien, Messenien vom Ende der myk. Palastzeit bis zur Einwanderung der Dorier (erscheint 1998) - K. FAGERSTröM, Greek Iron Age Architecture, $1988 \cdot$ R.HäGG, Die Gräber der Argolis in 
submyk., protogeom. und geom. Zeit, 1974 - R.HÄGG, N. Marinatos (Hrsg.), Sanctuaries and Cults in the Aegean Bronze Age, 198I - R.HäGg, N. Marinatos, G.C. NordQUist (Hrsg.), Early Greek Cult Practice, 1988 • V. Karageorghis (Hrsg.), Proceedings of the International Symposium "Cyprus in the 11 th century B. C. «, $1994^{\circ}$ G. KopCKE, Handel, in: ArchHom, M, 1990 - J. Latacz (Hrsg.), Zweihundert Jahre Homer-Forsch., Colloquium Rauricum Bd. 2, i99I - A. Mazarakis-Ainian, Late Bronze Age Apsidal and Oval Buildings in Greece and Adjacent Areas, in: A BSA 84, 1989, 269-288 - W. A. McDonald, W.D.E. Coulson, Excavations at Nichoria in Southwest Greece III, 1983 - D. Mustr u. a. (Hrsg.), La transizione dal Miceneo all'alto arcaismo, I99I - M.R. Popham et al. (Hrsg.), Lefkandi II/2, 1993 ' L.H. SACKETT, P. G. Themelis (Hrsg.), Lefkandi I. The Iron Age, $1979 / 1980$ - M. L. WeSt, The Rise of the Greek Epic, in: JHS I08, 1988, ISI-I72.

Karten-Lit.: V.R.D'A. Desborough, The Greek Dark Ages, $1972 \cdot$ F. SchachermeYr, Die ägäische Frühzeit, Bd. III, I979; IV, 1980 • G. KopKE, Handel, ArchHom, Kap. M, I990, bes. $78 \cdot$ J. VANSChOONWINKEL, L'Egée et la Mediterranée orientale à la fin du deuxième millénaire, I99 I.

\section{[2] (500-800 n. Chr.) s. Textgeschichte}

Duodecim scripta. Spiel, bei dem man versuchte, auf einem Brett die eigenen is Steine über das Ende der anderen Seite zu bringen. Mit zwei oder drei Würfeln bestimmte man die eigenen Züge; befanden sich auf einer Linie zwei oder drei Steine des Gegners, konnte auf diese Linie nicht gesetzt werden; war dort aber nur ein Stein, konnte man ihn entfernen. Nach Isid. orig. I 8,60 spielte man $d$. s. mit Spielturm, Würfel und Spielsteinen. Das Spielfeld bestand aus 36 Feldern, die mit geom. Figuren wie Kreisen oder Quadraten, Buchstaben(-gruppen) und Sinnsprüchen versehen waren. Spieltürme und -felder (bes. als Ritzungen in Steinpflasterungen) sind erh., ferner Spielszenen in der Kunst.

\section{$\rightarrow$ Brettspiele}

H. LAmer, s. v. Lusoria Tabula, RE I 3, 1979-198 s J.VÁterlein, Roma Ludens, in: Heuremata S, 1976, 55-57 - H. G. Horn, Si per me misit, nil nisi vota feret. Zu einem Spielturm aus Froitzheim, in. BJ I 89, I 989, 149-I 54

R.H.

Duoviri, Duumviri ("Zwei-Männer[-Amt]«; Sg. -dumum viru, daher auch "dummviriu) ist die Bezeichnung für verschiedenartige, histor. überlieferte Ämter mit Zweierbesetzung. Manche dieser Kollegien treten nur oder vor allem in bestimmten Epochen der röm. Republik auf.

D. perduellionis sind die seit früher republikanischer Zeit, im I. Jh.v. Chr. kaum noch eingesetzten Richter in Hochverratssachen (Liv. I,26,5f.; Cic. Rab. perd. I 2 f.).

D. sacris faciundis sind das Kollegium, dem im 4.Jh. v. Chr. die Befragung der Sibyllinischen Bücher über- tragen worden ist; später durch Xviri und dann durch XVviri s.f. ersetzt (Liv. 3, 10,7; Cic. fam. 8,4, I; Varro ling. 7,88).

D. agris dandis assignandis (au ch andere Mitgliederzahl möglich) sind Bevollmächtigte des röm. Staates für die Landverteilung an röm. Kolonisten (lex agr. 28 und $52 \mathrm{ff}$./FIRA I, IO2 ff., I09, I I 3).

D. navales sind die im 4. und 3. Jh.v. Chr. gelegentlich für Flottenbau und auch Flottenführung eingesetzten Bevollmächtigten (Liv. 9,30,4; 4I, I,3).

D. aedi dedicandae, faciundae, locandae sind beauf tragt, für Bau, Verwaltung und Nutzung von Tempeln zu sorgen (Liv. 7,28,5); bis zum Beginn der Kaiserzeit nachweisbar; dann übernehmen bes. procuratores ihre Aufgaben (Cass. Dio 55, 10,6).

Weitere Kollegien können für Spezialaufgaben gebildet werden, etwa die $d$. viis purgandis für die Reinhaltung von Straßen (tabula Heracleensis 5 I/FIRA I, I 40I 52) oder die $d$. aquae perducendae für die Wasserversorgung (Frontin. aqu. I,6; Cass. Dio 46,45,4).

Die bekannteste Form der $d$. ist das Spitzenamt der röm.-rechtlich verfaßten municipia und coloniae. Soweit an deren Spitze nicht squattuoniri et aediles (lex municipii Tarentini 9,2, I.H. des I.Jh. v. Chr., FIRA I, I 67), ein $\rightarrow$ pracfectus (lex de Gallia cisalpina $20 f f$. (um 50 v. Chr., FIRA I, I 69-I 75) oder ein Staatskommissar $(\rightarrow$ curatorrei publicae) stehen, liegt die Stadtregierung jeweils bei $d$. In einigen (latinischen) Städten besteht das Kollegium schon vor ihrer Unterordnung unter Rom, in anderen, bes. den coloniae, ist es als Kopie des stadtröm. Consulats zu erklären. Wie die histor. Entwicklung städtischer Ordnungen in coloniae und mmicipia, so ist auch die Funktion der $d$. in diesen vor dem I. Jh. v. Chr. nur in allg. Zügen bekannt; neben den $d$. gibt es wohl auch andere ortsspezifische Formen. Seit dem I. Jh.v. Chr. finden sich genauere Hinweise zu Aufgaben und Rechten der $d$. in einigen leges datae wie der lex municipii $\mathrm{Ma-}$ lacitani ( 52 v. Chr.; FIRA I, 202-219), der lex coloniae Genetivae Ursonensis (44 v. Chr.; FIRA I, 177-199) und der lex municipii Salpensani (82 n. Chr.; FIRA I, 202-208) sowie in Juristenschrif ten über das officium des proconsul. Als d. iure dicundo sind sie Beurkundungsbehörde und üben die Rechtssprechung (iurisdictio) auf unterer Ebene aus, ohne wie der röm. consul oder praetor ein imperium zu besitzen. Dieses liegt in der Prov. beim Statthalter (Dig. 50,1,26), welcher durch Edikt über alle die lex municipalis betref fenden Fragen entscheidet. Die $d$. berufen den ordo decurionmm und die Versammlung des Volkes ein, leiten die Wahl anderer Amtsträger und Funktionäre (vgl. lex municipii Malacitani 52; Dig. 50,4,14 und I8) und vertreten die Stadt beim Kaiser, bei staatlichen Stellen und anderen Städten auch rechtlich (Dig. 3,4,I und $6 ; 44,7,35,1$ ). Mit diesen Kompetenzen besteht das Kollegium bis in die Spätantike. Die $d$. werden auch zu dieser Zeit entweder vom Stadtvolk (Cod. Theod. I 2,5,I) oder von den städtischen dekuriones (Cod. Iust. I0,31,46) aus ihren Reihen (Dig. 50,2,7,2) gewählt. 